Korean J. Math. 19 (2011), No. 4, pp. 437-451

\title{
ON THE STABILITY OF THE GENERALIZED QUADRATIC AND ADDITIVE FUNCTIONAL EQUATION IN RANDOM NORMED SPACES VIA FIXED POINT METHOD
}

\author{
Sun SOOK JIN AND YANG-Hi LEE*
}

ABstract. In this paper, we prove the stability in random normed spaces via fixed point method for the functional equation

$$
f(x+2 y)-2 f(x+y)+2 f(x-y)-f(x-2 y)=0 .
$$

\section{Introduction}

In 1940, S. M. Ulam [20] raised a question concerning the stability of homomorphisms: Given a group $G_{1}$, a metric group $G_{2}$ with the metric $d(\cdot, \cdot)$, and a positive number $\varepsilon$, does there exist a $\delta>0$ such that if a mapping $f: G_{1} \rightarrow G_{2}$ satisfies the inequality

$$
d(f(x y), f(x) f(y))<\delta
$$

for all $x, y \in G_{1}$ then there exists a homomorphism $F: G_{1} \rightarrow G_{2}$ with

$$
d(f(x), F(x))<\varepsilon
$$

for all $x \in G_{1}$ ? As mentioned above, when this problem has a solution, we say that the homomorphisms from $G_{1}$ to $G_{2}$ are stable. In 1941, D. H. Hyers [5] gave a partial solution of Ulam's problem for the case of approximate additive mappings under the assumption that $G_{1}$ and $G_{2}$ are Banach spaces. Hyers' result was generalized by T. Aoki [1]

Received October 27, 2011. Revised December 12, 2011. Accepted December $14,2011$.

2000 Mathematics Subject Classification: 54E40, 39B82, 46S50, 46 S40.

Key words and phrases: stability, additive mapping, generalized quadratic and additive functional equation, random normed space.

*Corresponding author 
for additive mappings and Th. M. Rassias [16] for linear mappings by considering the stability problem with unbounded Cauchy differences. The paper of Th. M. Rassias has provided a lot of influence in the development of stability problems. The terminology Hyers-Ulam-Rassias stability originated from these historical background. During the last decades, the stability problems of functional equations have been extensively investigated by a number of mathematicians, see [2]-[4], [8], [9]-[12].

Recall, almost all subsequent proofs in this very active area have used Hyers' method, called a direct method. Namely, the function $F$, which is the solution of a functional equation, is explicitly constructed, starting from the given function $f$, by the formulae $F(x)=$ $\lim _{n \rightarrow \infty} \frac{1}{2^{n}} f\left(2^{n} x\right)$ or $F(x)=\lim _{n \rightarrow \infty} 2^{n} f\left(\frac{x}{2^{n}}\right)$. In 2003, V. Radu [15] observed that the existence of the solution $F$ of a functional equation and the estimation of the difference with the given function $f$ can be obtained from the fixed point alternative. In 2008, D. Mihet and V. Radu [14] applied this method to prove the stability theorems of the Cauchy functional equation:

$$
f(x+y)-f(x)-f(y)=0
$$

in random normed spaces. We call solutions of (1.1) additive maps. Recently, Jun and Kim [6,7] established the general solution and investigated the stability of the generalized quadratic and additive functional equation:

$$
f(x+2 y)-2 f(x+y)+2 f(x-y)-f(x-2 y)=0
$$

by using the direct method.

In this paper, using the fixed point method, we prove the stability for the functional equation (1.2) in random normed spaces. It is easy to see that the mapping $f(x)=a x^{2}+b x+c$ is a solution of (1.2). Every solution of the generalized quadratic and additive functional equation (1.2) is said to be a general quadratic mapping.

\section{Preliminaries}

In this section, we state the usual terminology, notations and conventions of the theory of random normed spaces, as in [18,19]. Firstly, 
the space of all probability distribution functions is denoted by $\Delta^{+}$

$:=\{F: \mathbb{R} \cup\{-\infty, \infty\} \rightarrow[0,1] \mid \mathrm{F}$ is left-continuous and nondecreasing on $\mathbb{R}$, where $F(0)=0$ and $F(+\infty)=1\}$.

And let the subset $D^{+} \subseteq \Delta^{+}$be the set $D^{+}:=\left\{F \in \Delta^{+} \mid l^{-} F(+\infty)=\right.$ 1 , where $l^{-} f(x)$ denotes the left limit of the function $f$ at the point $x$. The space $\Delta^{+}$is partially ordered by the usual pointwise ordering of functions, that is, $F \leq G$ if and only if $F(t) \leq G(t)$ for all $t \in \mathbb{R}$. The maximal element for $\Delta^{+}$in this order is the distribution function $\varepsilon_{0}: \mathbb{R} \cup\{0\} \rightarrow[0, \infty)$ given by

$$
\varepsilon_{0}(t)= \begin{cases}0, & \text { if } t \leq 0 \\ 1, & \text { if } t>0\end{cases}
$$

Definition 2.1. ([18]) A mapping $\tau:[0,1] \times[0,1] \rightarrow[0,1]$ is called a continuous triangular norm (briefly, a continuous $t$-norm) if $\tau$ satisfies the following conditions:

(a) $\tau$ is commutative and associative;

(b) $\tau$ is continuous;

(c) $\tau(a, 1)=a$ for all $a \in[0,1]$;

(d) $\tau(a, b) \leq \tau(c, d)$ whenever $a \leq c$ and $b \leq d$ for all $a, b, c, d \in[0,1]$.

Typical examples of continuous $t$-norms are $\tau_{P}(a, b)=a b, \tau_{M}(a, b)=$ $\min (a, b)$ and $\tau_{L}(a, b)=\max (a+b-1,0)$.

Definition 2.2. ([19]) A random normed space (briefly, $R N$-space ) is a triple $(X, \Lambda, \tau)$, where $X$ is a vector space, $\tau$ is a continuous $t$-norm, and $\Lambda$ is a mapping from $X$ into $D^{+}$such that the following conditions hold:

(RN1) $\Lambda_{x}(t)=\varepsilon_{0}(t)$ for all $t>0$ if and only if $x=0$,

$(\mathrm{RN} 2) \Lambda_{\alpha x}(t)=\Lambda_{x}(t /|\alpha|)$ for all $x$ in $X, \alpha \neq 0$ and all $t \geq 0$,

$(\mathrm{RN} 3) \Lambda_{x+y}(t+s) \geq \tau\left(\Lambda_{x}(t), \Lambda_{y}(s)\right)$ for all $x, y \in X$ and all $t, s \geq 0$.

If $(X,\|\cdot\|)$ is a normed space, we can define a mapping $\Lambda: X \rightarrow D^{+}$ by

$$
\Lambda_{x}(t)=\frac{t}{t+\|x\|}
$$

for all $x \in X$ and $t>0$. Then $\left(X, \Lambda, \tau_{M}\right)$ is a random normed space, which is called the induced random normed space. 
Definition 2.3. Let $(X, \Lambda, \tau)$ be an $R N$-space.

(i) A sequence $\left\{x_{n}\right\}$ in $X$ is said to be convergent to a point $x \in X$ if, for every $t>0$ and $\varepsilon>0$, there exists a positive integer $N$ such that $\Lambda_{x_{n}-x}(t)>1-\varepsilon$ whenever $n \geq N$.

(ii) A sequence $\left\{x_{n}\right\}$ in $X$ is called a Cauchy sequence if, for every $t>0$ and $\varepsilon>0$, there exists a positive integer $N$ such that $\Lambda_{x_{n}-x_{m}}(t)>1-\varepsilon$ whenever $n \geq m \geq N$.

(iii) An $R N$-space $(X, \Lambda, \tau)$ is said to be complete if and only if every Cauchy sequence in $X$ is convergent to a point in $X$.

Theorem 2.4. ([18]) If $(X, \Lambda, \tau)$ is an $R N$-space and $\left\{x_{n}\right\}$ is a sequence such that $x_{n} \rightarrow x$, then $\lim _{n \rightarrow \infty} \Lambda_{x_{n}}(t)=\Lambda_{x}(t)$.

\section{On the stability of a generalized quadratic and additive functional equation in random normed spaces via fixed point method}

We recall the fundamental result in the fixed point theory.

Theorem 3.1. ([13] or [17]) Suppose that a complete generalized metric space $(X, d)$, which means that the metric $d$ may assume infinite values, and a strictly contractive mapping $J: X \rightarrow X$ with the Lipschitz constant $0<L<1$ are given. Then, for each given element $x \in X$, either

$$
d\left(J^{n} x, J^{n+1} x\right)=+\infty, \forall n \in \mathbb{N} \cup\{0\},
$$

or there exists a nonnegative integer $k$ such that:

(1) $d\left(J^{n} x, J^{n+1} x\right)<+\infty$ for all $n \geq k$;

(2) the sequence $\left\{J^{n} x\right\}$ is convergent to a fixed point $y^{*}$ of $J$;

(3) $y^{*}$ is the unique fixed point of $J$ in $Y:=\left\{y \in X, d\left(J^{k} x, y\right)<\right.$ $+\infty\}$;

(4) $d\left(y, y^{*}\right) \leq(1 /(1-L)) d(y, J y)$ for all $y \in Y$.

Let $X$ and $Y$ be vector spaces. We use the following abbreviation for a given mapping $f: X \rightarrow Y$ by

$$
D f(x, y):=f(x+2 y)-2 f(x+y)+2 f(x-y)-f(x-2 y)
$$

for all $x, y \in X$. 
Lemma 3.2. Suppose that $f: X \rightarrow Y$ is a mapping such that $D f(x, y)=0$ for all $x, y \in X \backslash\{0\}$, then $f$ is a general quadratic mapping.

Proof. By the definition, it is clear that $\operatorname{Df}(x, 0)=0$ for all $x \in X$. Moreover, we have that

$$
\begin{aligned}
D f(0, x)= & D f(2 x, 2 x)-D f(4 x, x)-2 D f(3 x, x) \\
& \quad-2 D f(2 x, x)-2 D f(x, x) \\
= & 0
\end{aligned}
$$

for all $x \in X \backslash\{0\}$, which implies that $D f(x, y)=0$ for all $x, y \in X$. $\square$

Now we will establish the stability for the functional equation (1.2) in random normed spaces via fixed point method.

Theorem 3.3. Let $X$ be a linear space, $\left(Z, \Lambda^{\prime}, \tau_{M}\right)$ be an $R N$-space, $\left(Y, \Lambda, \tau_{M}\right)$ be a complete $R N$-space and $\varphi:(X \backslash\{0\})^{2} \rightarrow Z$. Suppose that $\varphi$ satisfies one of the following conditions:

(i) $\Lambda_{\alpha \varphi(x, y)}^{\prime}(t) \leq \Lambda_{\varphi(2 x, 2 y)}^{\prime}(t)$ for some $0<\alpha<2$,

(ii) $\Lambda_{\varphi(2 x, 2 y)}^{\prime}(t) \leq \Lambda_{\alpha \varphi(x, y)}^{\prime}(t)$ for some $4<\alpha$

for all $x, y \in X$ and $t>0$. If $f: X \rightarrow Y$ is a mapping such that

$$
\Lambda_{D f(x, y)}(t) \geq \Lambda_{\varphi(x, y)}^{\prime}(t)
$$

for all $x, y \in X \backslash\{0\}$ and $t>0$, then there exists a unique general quadratic mapping $F: X \rightarrow Y$ such that

$$
\Lambda_{f(x)-F(x)}(t) \geq \begin{cases}M\left(x, \frac{2(2-\alpha) t}{11}\right) & \text { if } \varphi \text { satisfies (i), } \\ M\left(x, \frac{(\alpha-4) t}{7}\right) & \text { if } \varphi \text { satisfies (ii) }\end{cases}
$$

for all $x \in X \backslash\{0\}$ and $t>0$, where

$$
\begin{aligned}
M(x, t) & =\tau_{M}\left\{\Lambda_{\varphi(2 x, 2 x)}^{\prime}(t), \Lambda_{\varphi(4 x, x)}^{\prime}(t), \Lambda_{\varphi(3 x, x)}^{\prime}(t), \Lambda_{\varphi(2 x, x)}^{\prime}(t), \Lambda_{\varphi(x, x)}^{\prime}(t),\right. \\
& \Lambda_{\varphi(-2 x,-2 x)}^{\prime}(t), \Lambda_{\varphi(-4 x,-x)}^{\prime}(t), \Lambda_{\varphi(-3 x,-x)}^{\prime}(t), \Lambda_{\varphi(-2 x,-x)}^{\prime}(t), \\
& \left.\Lambda_{\varphi(-x,-x)}^{\prime}(t), \Lambda_{\varphi\left(\frac{x}{2}, \frac{x}{2}\right)}^{\prime}(t), \Lambda_{\varphi\left(x, \frac{x}{2}\right)}^{\prime}, \Lambda_{\varphi\left(-\frac{x}{2},-\frac{x}{2}\right)}^{\prime}(t), \Lambda_{\varphi\left(-x,-\frac{x}{2}\right)}^{\prime}(t)\right\} .
\end{aligned}
$$

Moreover if $\alpha<1$ and $\Lambda_{\varphi(x, y)}^{\prime}$ is continuous in $x, y \in X \backslash\{0\}$ under the condition (i), then $f$ is a general quadratic mapping. 
Proof. Since $D g(x, y)=D f(x, y)$ for the mapping $g$ defined by $g(x)=f(x)-f(0)$, we can assume that $f(0)=0$ without loss of the generality. We will prove the theorem in two cases, $\varphi$ satisfies the condition (i) or (ii).

Case 1. Assume that $\varphi$ satisfies the condition (i). Let $S$ be the set of all functions $g: X \rightarrow Y$ with $g(0)=0$ and introduce a generalized metric on $S$ by

$d(g, h):=\inf \left\{u \in \mathbb{R}^{+} \mid \Lambda_{g(x)-h(x)}(u t) \geq M(x, t)\right.$ for all $\left.x \in X \backslash\{0\}\right\}$.

Consider the mapping $J: S \rightarrow S$ defined by

$$
J f(x):=\frac{f(2 x)-f(-2 x)}{4}+\frac{f(2 x)+f(-2 x)}{8}
$$

then we have

$$
J^{n} f(x)=\frac{1}{2}\left(4^{-n}\left(f\left(2^{n} x\right)+f\left(-2^{n} x\right)\right)+2^{-n}\left(f\left(2^{n} x\right)-f\left(-2^{n} x\right)\right)\right)
$$

for all $x \in X$. Let $f, g \in S$ and let $u \in[0, \infty]$ be an arbitrary constant with $d(g, f) \leq u$. From the definition of $d,(\mathrm{RN} 2)$, and (RN3), for the given $0<\alpha<2$ we have

$$
\begin{aligned}
& \Lambda_{J g(x)-J f(x)}\left(\frac{\alpha u}{2} t\right) \\
& =\Lambda_{\frac{3(g(2 x)-f(2 x))}{8}-\frac{g(-2 x)-f(-2 x)}{8}}\left(\frac{\alpha u}{2} t\right) \\
& \geq \tau_{M}\left\{\Lambda_{\frac{3(g(2 x)-f(2 x))}{8}}\left(\frac{3 \alpha u t}{8}\right), \Lambda_{\frac{g(-2 x)-f(-2 x)}{8}}\left(\frac{\alpha u t}{8}\right)\right\} \\
& \geq \tau_{M}\left\{\Lambda_{g(2 x)-f(2 x)}(\alpha u t), \Lambda_{g(-2 x)-f(-2 x)}(\alpha u t)\right\} \\
& \geq M(2 x, \alpha t) \\
& \geq M(x, t)
\end{aligned}
$$

for all $x \in X \backslash\{0\}$, which implies that

$$
d(J f, J g) \leq \frac{\alpha}{2} d(f, g) .
$$


That is, $J$ is a strictly contractive self-mapping of $S$ with the Lipschitz constant $\frac{\alpha}{2}$. Since the equality

$$
\begin{aligned}
& f(x)-J f(x) \\
& =\frac{1}{8}(-D f(2 x, 2 x)+D f(4 x, x)+2 D f(3 x, x)+2 D f(2 x, x) \\
& +2 D f(x, x)+D f(-2 x,-2 x)-D f(-4 x,-x)-2 D f(-3 x,-x) \\
& -2 D f(-2 x,-x)-2 D f(-x,-x)-2 D f\left(\frac{x}{2}, \frac{x}{2}\right) \\
& \left.-D f\left(x, \frac{x}{2}\right)-2 D f\left(-\frac{x}{2},-\frac{x}{2}\right)-D f\left(-x,-\frac{x}{2}\right)\right)
\end{aligned}
$$

holds for all $x \in X \backslash\{0\}$, by (3.1), we see that

$$
\begin{aligned}
& \Lambda_{f(x)-J f(x)}\left(\frac{11 t}{4}\right) \\
& \geq \tau_{M}\left\{\Lambda_{\frac{D f(2 x, 2 x)}{8}}\left(\frac{t}{8}\right), \Lambda_{\frac{D f(4 x, x)}{8}}\left(\frac{t}{8}\right), \Lambda_{\frac{D f(3 x, x)}{4}}\left(\frac{t}{4}\right),\right. \\
& \Lambda_{\frac{D f(2 x, x)}{4}}\left(\frac{t}{4}\right), \Lambda_{\frac{D f(x, x)}{4}}\left(\frac{t}{4}\right), \Lambda_{\frac{D f(-2 x,-2 x)}{8}}\left(\frac{t}{8}\right) \\
& \Lambda_{\frac{D f(-4 x,-x)}{8}}\left(\frac{t}{8}\right), \Lambda_{\frac{D f(-3 x,-x)}{4}}\left(\frac{t}{4}\right), \Lambda_{\frac{D f(-2 x,-x)}{4}}\left(\frac{t}{4}\right) \\
& \Lambda_{\frac{D f(-x,-x)}{4}}\left(\frac{t}{4}\right), \Lambda_{\frac{D f\left(\frac{x}{2}, \frac{x}{2}\right)}{4}}\left(\frac{t}{4}\right), \Lambda_{\frac{D f\left(x, \frac{x}{2}\right)}{8}}\left(\frac{t}{8}\right) \\
& \left.\Lambda_{\frac{D f\left(-\frac{x}{2},-\frac{x}{2}\right)}{4}}\left(\frac{t}{4}\right), \Lambda_{\frac{D f\left(-x,-\frac{x}{2}\right)}{8}}\left(\frac{t}{8}\right)\right\} \\
& \geq M(x, t)
\end{aligned}
$$

for all $x \in X \backslash\{0\}$. It means that $d(f, J f) \leq \frac{11}{4}<\infty$ by the definition of $d$. Therefore according to Theorem 3.1, the sequence $\left\{J^{n} f\right\}$ converges to the unique fixed point $F: X \rightarrow Y$ of $J$ in the set $T=\{g \in$ $S \mid d(f, g)<\infty\}$, which is represented by

$$
F(x):=\lim _{n \rightarrow \infty}\left(\frac{f\left(2^{n} x\right)+f\left(-2^{n} x\right)}{2 \cdot 4^{n}}+\frac{f\left(2^{n} x\right)-f\left(-2^{n} x\right)}{2^{n+1}}\right)
$$


for all $x \in X$. Since

$$
d(f, F) \leq \frac{1}{1-\frac{\alpha}{2}} d(f, J f) \leq \frac{11}{2(2-\alpha)}
$$

the inequality (3.2) holds. Next we will show that $F$ is a general quadratic mapping. Let $x, y \in X \backslash\{0\}$. Then by (RN3) we have

$$
\begin{aligned}
\Lambda_{D F(x, y)}(t) \geq \tau_{M}\{ & \Lambda_{\left(F-J^{n} f\right)(x+2 y)}\left(\frac{t}{8}\right), \Lambda_{2\left(J^{n} f-F\right)(x+y)}\left(\frac{t}{8}\right), \\
& \Lambda_{2\left(F-J^{n} f\right)(x-y)}\left(\frac{t}{8}\right), \Lambda_{\left(J^{n} f-F\right)(x-2 y)}\left(\frac{t}{8}\right), \\
& \left.\Lambda_{D J^{n} f(x, y)}\left(\frac{t}{2}\right)\right\}
\end{aligned}
$$

for all $n \in \mathbb{N}$. The first four terms on the right hand side of the above inequality tend to 1 as $n \rightarrow \infty$ by the definition of $F$. Now consider that

$$
\begin{aligned}
& \Lambda_{D J^{n} f(x, y)}\left(\frac{t}{2}\right) \geq \tau_{M}\left\{\Lambda_{\frac{D f\left(2^{n} x, 2^{n} y\right)}{2 \cdot 4^{n}}}\left(\frac{t}{8}\right), \Lambda_{\frac{D f\left(-2^{n} x,-2^{n} y\right)}{2 \cdot 4^{n}}}\left(\frac{t}{8}\right)\right. \\
& \left.\Lambda_{\frac{D f\left(2^{n} x, 2^{n} y\right)}{2 \cdot 2^{n}}}\left(\frac{t}{8}\right), \Lambda_{\frac{D f\left(-2^{n} x,-2^{n} y\right)}{2 \cdot 2^{n}}}\left(\frac{t}{8}\right)\right\} \\
& \geq \tau_{M}\left\{\Lambda_{D f\left(2^{n} x, 2^{n} y\right)}\left(\frac{4^{n} t}{4}\right), \Lambda_{D f\left(-2^{n} x,-2^{n} y\right)}\left(\frac{4^{n} t}{4}\right),\right. \\
& \left.\Lambda_{D f\left(2^{n} x, 2^{n} y\right)}\left(\frac{2^{n} t}{4}\right), \Lambda_{D f\left(-2^{n} x,-2^{n} y\right)}\left(\frac{2^{n} t}{4}\right)\right\} \\
& \geq \tau_{M}\left\{\Lambda_{\varphi(x, y)}^{\prime}\left(\frac{4^{n} t}{4 \alpha^{n}}\right), \Lambda_{\varphi(-x,-y)}^{\prime}\left(\frac{4^{n} t}{4 \alpha^{n}}\right),\right. \\
& \left.\Lambda_{\varphi(x, y)}^{\prime}\left(\frac{2^{n} t}{4 \alpha^{n}}\right), \Lambda_{\varphi(-x,-y)}^{\prime}\left(\frac{2^{n} t}{4 \alpha^{n}}\right)\right\}
\end{aligned}
$$

which tends to 1 as $n \rightarrow \infty$ by (RN3) and $\frac{2}{\alpha}>1$ for all $x, y \in X \backslash\{0\}$. Therefore it follows from (3.3) that

$$
\Lambda_{D F(x, y)}(t)=1
$$


for each $x, y \in X \backslash\{0\}$ and $t>0$. By (RN1) and Lemma 3.2, this means that $D F(x, y)=0$ for all $x, y \in X$. Assume that $0<\alpha<1$ and $\Lambda_{\varphi(x, y)}^{\prime}$ is continuous in $x, y \in X \backslash\{0\}$. If $m, a, b, c, d$ are any fixed integers with $a, c \neq 0$, then we have

$$
\begin{aligned}
\lim _{n \rightarrow \infty} \Lambda_{\varphi\left(\left(2^{n} a+b\right) x,\left(2^{n} c+d\right) y\right)}^{\prime}(t) & \geq \lim _{n \rightarrow \infty} \Lambda_{\varphi\left(\left(a+\frac{b}{2^{n}}\right) x,\left(c+\frac{d}{2^{n}}\right) y\right)}^{\prime}\left(\frac{t}{\alpha^{n}}\right) \\
& =\lim _{n \rightarrow \infty} \Lambda_{\varphi\left(\left(a+\frac{b}{2^{n}}\right) x,\left(c+\frac{d}{2^{n}}\right) y\right)}^{\prime}(m t) \\
& =\Lambda_{\varphi(a x, c y)}^{\prime}(m t)
\end{aligned}
$$

for all $x, y \in X \backslash\{0\}$ and $t>0$. Since $m$ is arbitrary, we have

$$
\lim _{n \rightarrow \infty} \Lambda_{\varphi\left(\left(2^{n} a+b\right) x,\left(2^{n} c+d\right) y\right)}^{\prime}(t) \geq \lim _{m \rightarrow \infty} \Lambda_{\varphi(a x, c y)}^{\prime}(m t)=1
$$

for all $x, y \in X \backslash\{0\}$ and $t>0$. From these, we get the inequality

$$
\begin{aligned}
& \Lambda_{2(F-f)(x)}(45 t) \\
& \geq \lim _{n \rightarrow \infty} \tau_{M}\left\{\Lambda_{(F-f)\left(\left(-2^{n}+1\right) x\right)}(11 t), \Lambda_{2(F-f)\left(\left(2^{n+1}+1\right) x\right)}(22 t),\right. \\
& \left.\quad \Lambda_{(f-F)\left(\left(3 \cdot 2^{n}+1\right) x\right)}(11 t), \Lambda_{(D f-D F)\left(\left(2^{n}+1\right) x,-2^{n} x\right)}(t)\right\} \\
& \geq \lim _{n \rightarrow \infty} \tau_{M}\left\{M\left(\left(-2^{n}+1\right) x, 2(2-\alpha) t\right), M\left(\left(2^{n+1}+1\right) x, 2(2-\alpha) t\right),\right. \\
& \left.\quad M\left(\left(3 \cdot 2^{n}+1\right) x, 2(2-\alpha) t\right), \Lambda_{\varphi\left(\left(2^{n}+1\right) x,-2^{n} x\right)}^{\prime}(t)\right\} \\
& =1
\end{aligned}
$$

for all $x \in X \backslash\{0\}$. From the above equality and the fact $f(0)=0=$ $F(0)$, we obtain $f \equiv F$. This completes the proof of this case.

Case 2. We take $\alpha>4$ and assume that $\varphi$ satisfies the condition (ii). Let the set $(S, d)$ be as in the proof of the case 1 . Now we consider the mapping $J: S \rightarrow S$ defined by

$$
J g(x):=g\left(\frac{x}{2}\right)-g\left(-\frac{x}{2}\right)+2\left(g\left(\frac{x}{2}\right)+g\left(-\frac{x}{2}\right)\right)
$$

for all $g \in S$ and $x \in X$. Notice that

$$
J^{n} g(x)=2^{n-1}\left(g\left(\frac{x}{2^{n}}\right)-g\left(-\frac{x}{2^{n}}\right)\right)+\frac{4^{n}}{2}\left(g\left(\frac{x}{2^{n}}\right)+g\left(-\frac{x}{2^{n}}\right)\right)
$$


for all $x \in X$ and $n \in \mathbb{N}$. Let $f, g \in S$ and let $u \in[0, \infty]$ be an arbitrary constant with $d(g, f) \leq u$. From the definition of $d,(\mathrm{RN} 2)$, and (RN3), we have

$$
\begin{aligned}
\Lambda_{J g(x)-J f(x)}\left(\frac{4 u}{\alpha} t\right) & =\Lambda_{3\left(g\left(\frac{x}{2}\right)-f\left(\frac{x}{2}\right)\right)+g\left(-\frac{x}{2}\right)-f\left(-\frac{x}{2}\right)}\left(\frac{4 u}{\alpha} t\right) \\
& \geq \tau_{M}\left\{\Lambda_{3\left(g\left(\frac{x}{2}\right)-f\left(\frac{x}{2}\right)\right)}\left(\frac{3 u}{\alpha} t\right), \Lambda_{g\left(-\frac{x}{2}\right)-f\left(-\frac{x}{2}\right)}\left(\frac{u}{\alpha} t\right)\right\} \\
& \geq \tau_{M}\left\{\Lambda_{g\left(\frac{x}{2}\right)-f\left(\frac{x}{2}\right)}\left(\frac{u}{\alpha} t\right), \Lambda_{g\left(-\frac{x}{2}\right)-f\left(-\frac{x}{2}\right)}\left(\frac{u}{\alpha} t\right)\right\} \\
& \geq M\left(\frac{x}{2}, \frac{t}{\alpha}\right) \\
& \geq M(x, t)
\end{aligned}
$$

for all $x \in X$, which implies that

$$
d(J f, J g) \leq \frac{4}{\alpha} d(f, g) .
$$

That is, $J$ is a strictly contractive self-mapping of $S$ with the Lipschitz constant $0<\frac{4}{\alpha}<1$. Since the equality

$$
\begin{aligned}
& f(2 x)-J f(2 x) \\
& =-\frac{1}{4}(-D f(2 x, 2 x)+D f(4 x, x)+2 D f(3 x, x)+2 D f(2 x, x) \\
& +2 D f(x, x)+D f(-2 x,-2 x)-D f(-4 x,-x)-2 D f(-3 x,-x) \\
& -2 D f(-2 x,-x)-2 D f(-x,-x)-4 D f\left(\frac{x}{2}, \frac{x}{2}\right) \\
& \left.-2 D f\left(x, \frac{x}{2}\right)-4 D f\left(-\frac{x}{2},-\frac{x}{2}\right)-2 D f\left(-x,-\frac{x}{2}\right)\right)
\end{aligned}
$$


holds for all $x \in X \backslash\{0\}$, we get

$$
\begin{aligned}
\Lambda_{f(2 x)-J f(2 x)}(7 t) & \geq \tau_{M}\left\{\Lambda_{\frac{D f(2 x, 2 x)}{4}}\left(\frac{t}{4}\right), \Lambda_{\frac{D f(4 x, x)}{4}}\left(\frac{t}{4}\right), \Lambda_{\frac{D f(3 x, x)}{2}}\left(\frac{t}{2}\right),\right. \\
& \Lambda_{\frac{D f(2 x, x)}{2}}\left(\frac{t}{2}\right), \Lambda_{\frac{D f(x, x)}{2}}\left(\frac{t}{2}\right), \Lambda_{\frac{D f(-2 x,-2 x)}{4}}\left(\frac{t}{4}\right) \\
& \Lambda_{\frac{D f(-4 x,-x)}{4}}\left(\frac{t}{4}\right), \Lambda_{\frac{D f(-3 x,-x)}{2}}\left(\frac{t}{2}\right), \Lambda_{\frac{D f(-2 x,-x)}{2}}\left(\frac{t}{2}\right) \\
& \Lambda_{\frac{D f(-x,-x)}{2}}\left(\frac{t}{2}\right), \Lambda_{D f\left(\frac{x}{2}, \frac{x}{2}\right)}(t), \Lambda_{\frac{D f\left(x, \frac{x}{2}\right)}{2}}\left(\frac{t}{2}\right) \\
& \left.\Lambda_{D f\left(-\frac{x}{2},-\frac{x}{2}\right)}(t), \Lambda_{\frac{D f\left(-x,-\frac{x}{2}\right)}{2}}\left(\frac{t}{2}\right)\right\} \\
& \geq M(x, t)
\end{aligned}
$$

for all $x \in X \backslash\{0\}$. From this, we have

$$
\Lambda_{f(x)-J f(x)}\left(\frac{7 t}{\alpha}\right) \geq M\left(\frac{x}{2}, \frac{t}{\alpha}\right) \geq M(x, t)
$$

for all $x \in X \backslash\{0\}$. It means that $d(f, J f) \leq \frac{7}{\alpha}<\infty$ by the definition of $d$. Therefore according to Theorem 3.1, the sequence $\left\{J^{n} f\right\}$ converges to the unique fixed point $F: X \rightarrow Y$ of $J$ in the set $T=\{g \in$ $S \mid d(f, g)<\infty\}$, which is represented by

$$
F(x):=\lim _{n \rightarrow \infty}\left(2^{n-1}\left(f\left(\frac{x}{2^{n}}\right)-f\left(-\frac{x}{2^{n}}\right)\right)+\frac{4^{n}}{2}\left(f\left(\frac{x}{2^{n}}\right)+f\left(-\frac{x}{2^{n}}\right)\right)\right)
$$

for all $x \in X$. Since

$$
d(f, F) \leq \frac{1}{1-\frac{4}{\alpha}} d(f, J f) \leq \frac{7}{\alpha-4}
$$

the inequality (3.2) holds. Next we will show that $F$ is a general quadratic . Recall that the inequality (3.3) holds for all $x, y \in X \backslash\{0\}$ 
and $n \in \mathbb{N}$. The first four terms on the right hand side of the inequality (3.3) tend to 1 as $n \rightarrow \infty$ by the definition of $F$. Now consider that

$$
\begin{aligned}
\Lambda_{D J^{n} f(x, y)}\left(\frac{t}{2}\right) \geq & \tau_{M}\left\{\Lambda_{2^{2 n-1} D f\left(\frac{x}{2^{n}}, \frac{y}{2^{n}}\right)}\left(\frac{t}{8}\right), \Lambda_{2^{2 n-1} D f\left(\frac{-x}{2^{n}}, \frac{-y}{2^{n}}\right)}\left(\frac{t}{8}\right)\right. \\
& \left.\Lambda_{2^{n-1} D f\left(\frac{x}{2^{n}}, \frac{y}{2^{n}}\right)}\left(\frac{t}{8}\right), \Lambda_{-2^{n-1} D f\left(\frac{-x}{2^{n}}, \frac{-y}{2^{n}}\right)}\left(\frac{t}{8}\right)\right\} \\
\geq & \tau_{M}\left\{\Lambda_{\varphi(x, y)}^{\prime}\left(\frac{\alpha^{n} t}{4^{n+1}}\right), \Lambda_{\varphi(-x,-y)}^{\prime}\left(\frac{\alpha^{n} t}{4^{n+1}}\right)\right. \\
& \left.\Lambda_{\varphi(x, y)}^{\prime}\left(\frac{\alpha^{n} t}{2^{n+2}}\right), \Lambda_{\varphi(-x,-y)}^{\prime}\left(\frac{\alpha^{n} t}{2^{n+2}}\right)\right\}
\end{aligned}
$$

which tends to 1 as $n \rightarrow \infty$ by (RN3) for all $x, y \in X \backslash\{0\}$. Therefore it follows from (3.3) that

$$
\Lambda_{D F(x, y)}(t)=1
$$

for each $x, y \in X \backslash\{0\}$ and $t>0$. By (RN1) and Lemma 3.2, this means that $D F(x, y)=0$ for all $x, y \in X$. It completes the proof of this theorem.

Now we have a generalized Hyers-Ulam stability of the general quadratic functional equation (1.2) in the framework of normed spaces. Let $\Lambda_{x}(t)=\frac{t}{t+\|x\|}$. Then $\left(X, \Lambda, \tau_{M}\right)$ is an induced random normed space, which leads us to get the following result.

Corollary 3.4. Let $X$ be a linear space, $Y$ be a complete normedspace, and $\varphi:(X \backslash\{0\})^{2} \rightarrow[0, \infty)$. Suppose that $\varphi$ satisfies one of the following conditions:

(i) $\alpha \varphi(x, y) \geq \varphi(2 x, 2 y)$ for some $0<\alpha<2$,

(ii) $\varphi(2 x, 2 y) \geq \alpha \varphi(x, y)$ for some $4<\alpha$

for all $x, y \in X \backslash\{0\}$. If $f: X \rightarrow Y$ is a mapping such that

$$
\|D f(x, y)\| \leq \varphi(x, y)
$$

for all $x, y \in X \backslash\{0\}$, then there exists a unique general quadratic mapping $F: X \rightarrow Y$ such that

$$
\|f(x)-F(x)\| \leq \begin{cases}\frac{11 \Phi(x)}{2(2-\alpha)} & \text { if } \varphi \text { satisfies (i) } \\ \frac{7 \Phi(x)}{\alpha-4} & \text { if } \varphi \text { satisfies (ii) }\end{cases}
$$


for all $x \in X \backslash\{0\}$, where $\Phi(x)$ is defined by

$$
\begin{aligned}
\Phi(x)=\max \{ & \varphi(2 x, 2 x), \varphi(4 x, x), \varphi(3 x, x), \varphi(2 x, x), \varphi(x, x), \varphi(-2 x,-2 x), \\
& \varphi(-4 x,-x), \varphi(-3 x,-x), 2 \varphi(-2 x,-x), \varphi(-x,-x), \\
& \left.\varphi\left(\frac{x}{2}, \frac{x}{2}\right), \varphi\left(x, \frac{x}{2}\right), \varphi\left(-\frac{x}{2},-\frac{x}{2}\right), \varphi\left(-x,-\frac{x}{2}\right)\right\} .
\end{aligned}
$$

Moreover, if $0<\alpha<1$ and $\varphi$ is continuous on $(X \backslash\{0\})^{2}$ under the condition (i), then $f$ is itself a general quadratic mapping.

Now we have Hyers-Ulam-Rassias stability results of the general quadratic functional equation (1.2).

Corollary 3.5. Let $X$ be a normed space, $p \in \mathbb{R} \backslash[1,2]$ and $Y$ a complete normed-space. If $f: X \rightarrow Y$ is a mapping such that

$$
\|D f(x, y)\| \leq\|x\|^{p}+\|y\|^{p}
$$

for all $x, y \in X \backslash\{0\}$, then there exists a unique general quadratic mapping $F: X \rightarrow Y$ such that

$$
\|f(x)-F(x)\| \leq \begin{cases}\frac{11\left(4^{p}+1\right)\|x\|^{p}}{2\left(2-2^{p}\right)} & \text { if } 0 \leq p<1, \\ \frac{7\left(4^{p}+1\right)\|x\|^{p}}{2^{p}-4} & \text { if } p>2\end{cases}
$$

for all $x \in X \backslash\{0\}$ and $f$ is itself a general quadratic mapping if $p<0$.

Proof. If we denote by $\varphi(x, y)=\|x\|^{p}+\|y\|^{p}$, then the induced random normed space $\left(X, \Lambda_{x}, \tau_{M}\right)$ holds the conditions of Corollary 3.4 with $\alpha=2^{p}$.

Corollary 3.6. Let $X$ be a normed space, $p+q \in \mathbb{R} \backslash[1,2]$ and $Y$ a complete normed-space. If $f: X \rightarrow Y$ is a mapping such that

$$
\|D f(x, y)\| \leq\|x\|^{p}\|y\|^{q}
$$

for all $x, y \in X \backslash\{0\}$, then there exists a unique general quadratic mapping $F: X \rightarrow Y$ such that

$$
\|f(x)-F(x)\| \leq \begin{cases}\frac{11 \cdot\|2 x\|^{p+q}}{2\left(2-2^{p+q}\right)} & \text { if } 0 \leq p+q<1 \text { and } p \leq q \\ \frac{11 \cdot 4^{p}\|x\|^{p+q}}{2\left(2-2^{p+q}\right)} & \text { if } 0 \leq p+q<1 \text { and } q \leq p \\ \frac{7 \cdot\|2 x\|^{p+q}}{2^{p}-4} & \text { if } p+q>2 \text { and } p \leq q \\ \frac{7 \cdot 4^{p}\|x\|^{p+q}}{\left(2^{p}-4\right) 2^{p}} & \text { if } p+q>2 \text { and } q \leq p\end{cases}
$$


for all $x \in X \backslash\{0\}$ and $f$ is itself a general quadratic mapping if $p+q<0$.

Proof. If we denote by $\varphi(x, y)=\|x\|^{p}\|y\|^{q}$, then the induced random normed space $\left(X, \Lambda_{x}, \tau_{M}\right)$ holds the conditions of Corollary 3.4 with $\alpha=2^{p+q}$.

\section{References}

[1] T. Aoki, On the stability of the linear transformation in Banach spaces, J. Math. Soc. Japan 2 (1950), 64-66.

[2] P.W. Cholewa, Remarks on the stability of functional equations, Aequationes Math. 27 (1984), 76-86.

[3] Z. Gajda, On stability of additive mappings, Int. J. Math. Math. Sci. 14 (1991), 431-434.

[4] P. Găvruta, A generalization of the Hyers-Ulam-Rassias stability of approximately additive mappings, J. Math. Anal. Appl. 184 (1994), 431-436.

[5] D.H. Hyers, On the stability of the linear functional equation, Proc. Natl. Acad. Sci. USA 27 (1941), 222-224.

[6] K.-W. Jun and H.-M. Kim, On the stability of a general quadratic functional equation and applications, J. Chungcheong Math. Soc. 17 (2004), 57-75.

[7] K.-W. Jun and H.-M. Kim, On the Hyers-Ulam stability of a generalized quadratic and additive functional equation, Bull. Korean Math. Soc. 42 (2005), 133-148.

[8] S.-M. Jung, On the Hyers-Ulam stability of the functional equations that have the quadratic property, J. Math. Anal. Appl. 222 (1998), 126-137.

[9] Y.-H. Lee, On the stability of the monomial functional equation, Bull. Korean Math. Soc. 45 (2008), 397-403.

[10] Y.-H. Lee and K.W. Jun, A generalization of the Hyers-Ulam-Rassias stability of Jensen's equation, J. Math. Anal. Appl. 238 (1999), 305-315.

[11] Y.-H. Lee and K.W. Jun, A generalization of the Hyers-Ulam-Rassias stability of Pexider equation, J. Math. Anal. Appl. 246 (2000), 627-638.

[12] Y.-H. Lee and K.W. Jun, On the stability of approximately additive mappings, Proc. Amer. Math. Soc. 128 (2000), 1361-1369.

[13] B. Margolis and J.B. Diaz, A fixed point theorem of the alternative for contractions on a generalized complete metric space, Bull. Amer. Math. Soc. 74 (1968), 305-309.

[14] D. Mihet and V. Radu, On the stability of the additive Cauchy functional equation in random normed spaces, J. Math. Anal. Appl. 343 (2008), 567-572.

[15] V. Radu, The fixed point alternative and the stability of functional equations, Fixed Point Theory 4 (2003), 91-96.

[16] Th.M. Rassias, On the stability of the linear mapping in Banach spaces, Proc. Amer. Math. Soc. 72 (1978), 297-300. 
[17]I.A. Rus, Principles and applications of fixed point theory (in Romanian), Ed. Dacia, Cluj-Napoca, 1979.

[18] B. Schweizer and A. Sklar, Probabilistic metric spaces, Elsevier, North Holand, New York, 1983.

[19] A.N. S̆erstnev, On the motion of a random normed space, Dokl. Akad. Nauk 149 (1963), 280-283.

[20] S.M. Ulam, A collection of mathematical problems, Interscience, New York, 1968.

Department of Mathematics Education

Gongju National University of Education

Gongju 314-711, Republic of Korea

E-mail: ssjin@gjue.ac.kr

Department of Mathematics Education

Gongju National University of Education

Gongju 314-711, Republic of Korea

E-mail: yanghi2@hanmail.net 